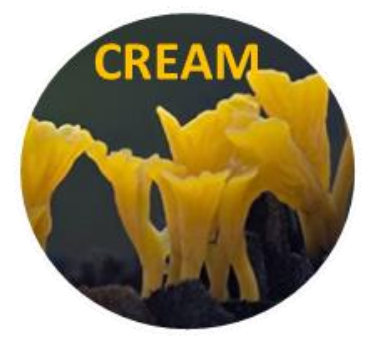

Current Research in Environmental \& Applied Mycology 6 (3): 166-172(2016) ISSN 2229-2225

www.creamjournal.org

Article

CREAM

Copyright $(2016$

Doi 10.5943/cream/6/3/3

Online Edition

\title{
An effective approach of strain improvement in Cordyceps militaris using abrin
}

\author{
Mani $\mathrm{A}^{1^{*}}$, Thawani $\mathbf{V}^{1}$ and Zaidi $\mathrm{KU}^{1}$
}

\begin{abstract}
${ }^{1}$ Biotechnology \& Pharmacology Laboratory, Centre for Scientific Research and Development, 4th floor, People's University Building People's University, Bhanpur, Bhopal 462037, Madhya Pradesh, India. Email: am.csrd@gmail.com
\end{abstract}

Mani A, Thawani V, Zaidi KU 2016 - An effective approach of strain improvement in Cordyceps militaris using abrin. Current Research in Environmental \& Applied Mycology 6(3), 166-172, Doi $10.5943 / \mathrm{cream} / 6 / 3 / 3$

\begin{abstract}
Cordyceps militaris, a medicinal fungus facing conservation from excessive harvesting and specific environment need for growth. In this study, three different techniques for strain improvement viz., somatic fusion, protoplast fusion and UV irradiation were assessed for biomass, exo-polymer and cordycepin production. It was observed that, somatic hybridization was more effective and the developed hybrids were stable even after sub culturing. Moreover hybrids developed by protoplast fusion were stable rather U-V irradiation mutant showed characteristic changes after sub culturing. In the study abrin was used to trigger somatic fusion and it was found to be an effective approach in strain improvement.
\end{abstract}

Key words - Biomass - Cordycepin - Exo-polymer - plasma fusion - somatic hybrization - UV irradiation

\section{Introduction}

Cordyceps militaris (Fr.) Link is an entomo-pathogenic fungus, which has been extensively studied for its medicinal uses. The myriad of its health benefits include immunomodulatory, hypoglycemic, hypolipidemic, and antitumor activity (Russell\& Paterson 2008, Zhou et al. 2009, Das et al. 2010, Lin \& Li 2011). C. militaris has been recently approved as a functional food and Traditional Chinese Medicine by Chinese government (Wen et al. 2014a). In the nature C. militarisis rare and expensive, since the fungus is host specific and requires strict growth environment, hence its commercial utilization is a tough issue (Patel \& Ingalhalli 2013). This study was directed at strain improvement for metabolite and biomass production. Protoplast fusion is a known to be a feasible method for inter-specific and inter-generic hybridization edible mushrooms (Aswini et al. 2014) but its yield is poor as compared to non-basidiomycetous fungi (Gupta et al. 1997). Hybridization and Protoplast fusion can produce hybrids with the combined properties of both parents (Zhao \& Chang 1995, Feng et al. 2012). Holliday et al. (2004) developed hybrid strains of $C$. sinensis using rattle snake venom by triggering somatic fusion which resulted in reproducible strain containing qualities of recognized bioactive compounds. The effect of UV light on vegetative compatibility in the fungus and developed the procedure to detect individual, incompatible colonies from cultured lawns of compatible colonies (Rizwana \& Powell 1992). Zhao et al. (2011) introduced inactivated protoplast fusion by UV 
irradiation in fungi. Mutants from these techniques proved to be more efficient than the original strain (Alberts et al. 2002).There are no studies conducted for strain improvement in $C$. militaris even though this fungus has good industrial application. This study focuses on the development of hybrids to produce more potent metabolite from the two strains of $C$. militaris.

\section{Materials and Methods}

\section{Micro-organism and Medium}

C. militaris (MTCC 3936) and C. militaris wild strain (CCM 1) was cultured in Corn Meal Agar (CMA) at $20^{\circ} \mathrm{C}$ for two weeks. For mycelia culturing Potato malt Peptone (PMP), medium pH 6.0 at $20^{\circ} \mathrm{C}$ on shaking condition was used for six days. Then the culture flasks were incubated at $20^{\circ} \mathrm{C}$ for 10-15 days, without shaking.

\section{Strain Development}

\section{Hybridization}

Abrin was extracted by soaking $10 \mathrm{~g}$ pulverized seeds of Abrus precatorius in PBS (pH 7.7) with $5 \% \mathrm{NaCl}$ for overnight at $4^{\circ} \mathrm{C}$. The homogenate was filtered and centrifuged at $10,000 \mathrm{rpm}$ for 30 min at $4^{\circ} \mathrm{C}$. The crude abrin was dialyzed against distilled water against $10 \mathrm{mM}$ Tris- $\mathrm{HC} 1 \mathrm{pH} 7.7$ for 24 $\mathrm{h}$ each. The dialyzed solution was centrifuged at 15,000 for $20 \mathrm{~min}$, and the partially purified abrin was used for hybridization.

Corn meal agar (CMA) plates were prepared and inoculated with two strains of $C$. militarisviz. MTCC 3936 and CCM-1, on opposite sides of the Petri-plate and were incubated at $20^{\circ} \mathrm{C}$. After one week of incubation, a furrow was made in the middle of the plate of $7 \mathrm{~cm} \mathrm{X} 0.2 \mathrm{~cm}$ and $100 \mu 1$ of crude abrin $\left(20 \mu \mathrm{g} / \mathrm{ml}\right.$ total protein) was added. The plates were again incubated at $20^{\circ} \mathrm{C}$ for more than one week till mycelial growth was seen, when the stromata were initiated at the meeting line between the strains, and were isolated into a new CMA.

\section{Protoplast fusion}

The spores were collected by centrifuging of filtered liquid culture at 10,000 rpm for $10 \mathrm{~min}$. The spore suspension of each parent was inoculated in $50 \mathrm{ml}$ protoplasting medium (Glucose $80 \mathrm{~g} / \mathrm{L}$; $\mathrm{NH}_{4} \mathrm{NO}_{3} 2 \mathrm{~g} / \mathrm{L} ; \mathrm{KH}_{2} \mathrm{PO}_{4} 10 \mathrm{~g} / \mathrm{L} ; \mathrm{MgSO}_{4} \cdot 7 \mathrm{H}_{2} \mathrm{O} 0.25 \mathrm{~g} / \mathrm{L} ; \mathrm{FeCl}_{3} \cdot 6 \mathrm{H}_{2} \mathrm{O} 0.02 \mathrm{~g} / \mathrm{L} ; \mathrm{MnSO}_{4} 0.014 \mathrm{~g} / \mathrm{L} ; \mathrm{pH}$ $4.5)$ and incubated for $36 \mathrm{hr}$ to $20^{\circ} \mathrm{C}$ on rotatory shaker $(180 \mathrm{rpm})$. The mycelium formed was recovered by centrifugation $12,000 \mathrm{rpm}$ for $10 \mathrm{~min}$, the mycelium was washed twice with sterilized physiological saline $(0.85 \% \mathrm{NaCl})$ and resuspended in $5 \mathrm{ml}$ protoplasting buffer $(0.1 \mathrm{M}$; pH $5.8-6)$ containing $0.7 \mathrm{M} \mathrm{NaCl} ; 0.2 \mathrm{M} \mathrm{CaCl}_{2}$ and Novozyme $234(20 \mathrm{mg} / \mathrm{ml})$ and incubated at $20^{\circ} \mathrm{C}$ with gentle shaking for up to $6 \mathrm{hr}$. Then equal volume of suspension from two parents were mixed and centrifuged. The protoplast pellet formed was resuspended in $2 \mathrm{ml}$ fusion buffer (Phosphate buffer $0.05 \mathrm{M} ; \mathrm{pH} 7.5$, $30 \% \mathrm{w} / \mathrm{v}$ PEG $6000,50 \mathrm{mM} \mathrm{CaCl} 2$ and $0.7 \mathrm{M} \mathrm{NaCl}$.) and incubated for $20 \mathrm{~min}$ at $20^{\circ} \mathrm{C}$ and the suspension was inoculated on CMA media.

\section{UV irradiation}

Sporulation was induced by growning strains on CMA plates at $20^{\circ} \mathrm{C}$ and subcultured. After subculturing, conidial suspension was prepared $0.85 \% \mathrm{NaCl}$ containing $0.1 \%$ Tween 80 . The suspension was treated with $500 \mathrm{mug} / \mathrm{ml}$ sodium nitrate and was irradiated of 80 min under UV lamp at the distance of $50 \mathrm{~cm}$. After irradiation the conidial suspension were incubated at $20^{\circ} \mathrm{C}$ for $60 \mathrm{~min}$ in dark. After incubation, $0.1 \mathrm{ml}$ was poured on solidified PMP medium to restrict the growth of fungal colonies. The plates were incubated at $20^{\circ} \mathrm{C}$ for six days until the fungal colonies were observed. Those isolates which retained the altered characters were identified as mutant. 


\section{Screening improved strains}

The strains were screened for biomass, exo-polymer and cordycepin production; all strains were inoculated in PMP pH6.0 and analyzed the production of mycelial biomass and exo-biopolymer, strains that produced maximum biomass and exo-biopolymer were selected for the further studies.

Disc measuring $1 \mathrm{~cm}$ diameter of selected cultures were inoculated into PMP mediums and inoculated at $20^{\circ} \mathrm{C}$ at $\mathrm{pH} 6.0$ for two weeks with initial four days under shaking. After cultivation in liquid PMP, the mycelial biomass was harvested by pelleting the culture at 10,000 rpm for 10 min. Dry weight of mycelia was recorded. The exo-polymer was extracted by concentrating the supernatant to $1 / 4$ th of its original volume and mixed with four times with absolute ethanol, stirred vigorously and then incubated overnight at $4{ }^{\circ} \mathrm{C}$ for precipitation (Bae et al. 2000). The cordycepin was extracted from the liquid phase by extracting sequentially from hexane, chloroform and butanol $(1: 10 \mathrm{w} / \mathrm{v}$ for all solvents) the butanol extract was further purified in silica gel column with chloroform: methanol in 9:1 ratio. Cordycepin was analyzed by HPLC according to Wen et al. (2014b).

\section{Result}

\section{Hybridization}

The growth of both strains MTCC-3936 and CCM-1 were inhibited by abrin for 3 days at the furrow region and after 3 days the mycelia started growing but the mycelia growth was slow. It was observed that at the meeting point the stroma was producing (Fig.1) and was able to grow faster that earlier. The 10 stromas were isolated from the meeting points into fresh CMA plates and inoculated at $20^{\circ} \mathrm{C}$ for two weeks. After incubation, disc of $1 \mathrm{~cm}$ from each plates were inoculated into PMP medium for evaluating the biomass and exo-polymer production. Four hybrid strains $\mathrm{H}-1, \mathrm{H}-2, \mathrm{H}-3$ and $\mathrm{H}-4$ (Fig. 2) were selected for future studies based on the high biomass production. To best of the knowledge the use of abrin to trigger somatic fusion is not yet reported. The study indicated that abrin is also an effective trigger to develop hybrids. The present study showed that $C$. militaris can withstand the inhibitory properties of abrin after somatic fusion and thus can be used as a good mode for effective hybridization since it inhibits protein synthesis of cells. The hybrids produced through this method have stability in biomass and exo-polymer production even after repeated sub culturing even after year storage (Data not given).
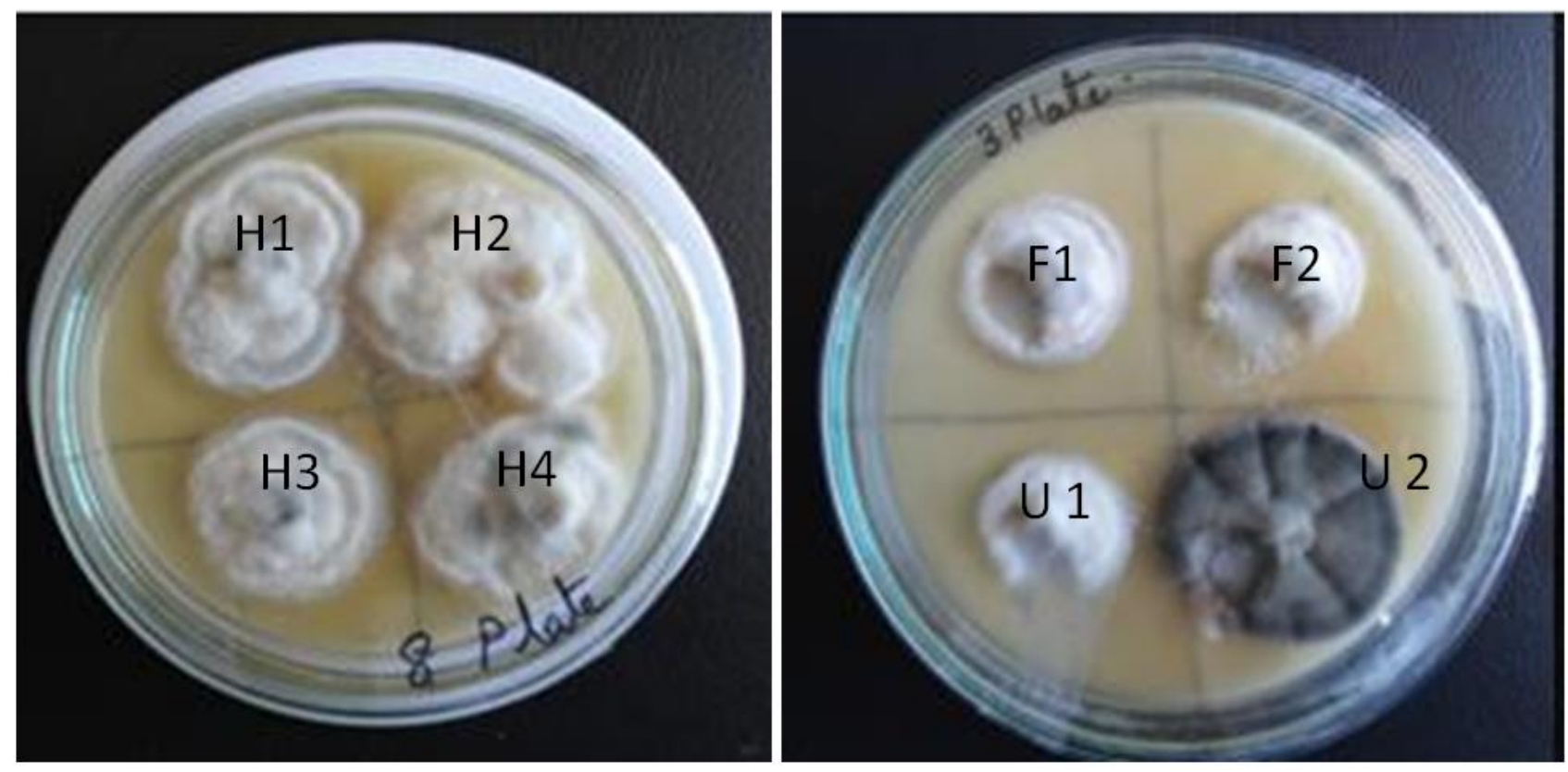
Fig. 1- Strains of C. militaris (MTCC-3936 (A) and CCM-1(B) in corn meal agar plate showing a growth inhibition in the presence of abrin and stroma development

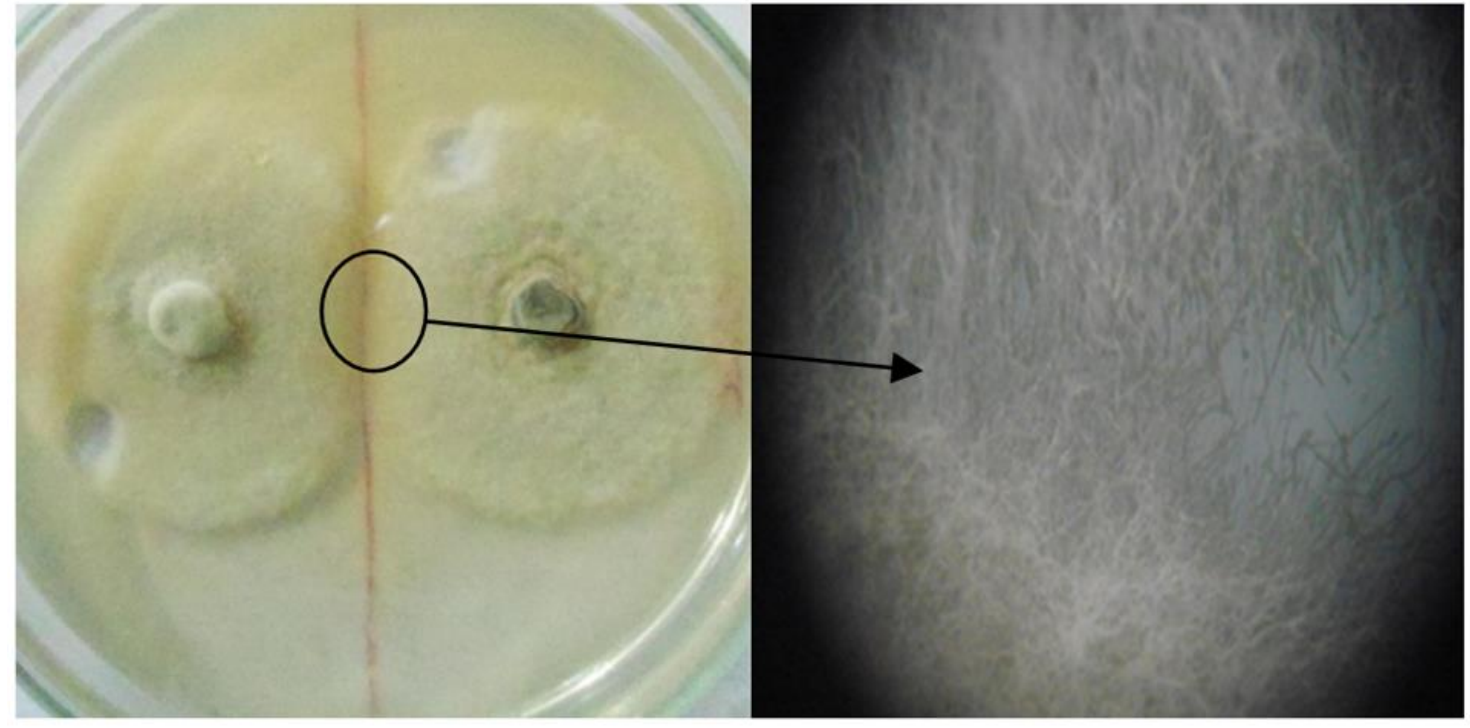

Fig. 2 - Improved $C$. militaris strains through hybridization (H1, H2, H3 and H4), Protoplast fusion (F1 and F2) and UV-irradiation (U1andU2)

\section{Protoplast fusion}

The re-suspended spores in protoplasting buffer were investigated for clumps under microscope. After fusion white colored strains of combination were observed were selected for further studies. Two fused cultures (F-1 and F-2, Fig. 2) were selected for comparative analysis based on high biomass production. It was observed that the strain were stable after sub culturing with similar biomass production rather decreased metabolites. It was also observed the strain were having combined characteristics both parents. The present study inactivation procedure using heat and UV was not followed to stabilize the fusion rather the fused suspension was directly inoculated to CMA from which the culture was isolated. It was observed that the isolates were stable even after sub-culturing.

\section{UV-irradiation}

After incubated for six to seven days, only an average of five colonies were observed viable and it was inoculated in $150 \mathrm{ml}$ of PMP broth and incubated for 10 days at $20^{\circ} \mathrm{C}$. After incubation the culture was grown in CMA plates (Fig 2). U-1 (MTCC-3936), U-2 (CCM-1) from each parents were selected for further analysis. It was observed that the U-2 isolate showed change in it colony color from white to black colony (Fig. 2). More over the U-1 was having slow growth indicated mutation. It was observed that by this method the harvest of live spores and the viability of the cells were less. It was also observed that after repeated sub culturing the mutant colony were losing its characteristics and viability.

\section{Characterization of Hybrids}

There was a higher biomass production in the hybrids as considered parental culture (MTCC3936 and CCM) rather the cultured developed through plasma fusion where having similar parental characteristics. It was observed that the UV treated culture had morphological differences; U1 mutant had less mycelia biomass (0.86 g/L, Dry weight). Moreover the U-2 mutant developed from CCM culture had triggered mycelia growth than the parental strain. The three methods were effective in triggering the metabolic production and mycelia growth (Fig. 3). It was observed that, the hybrid H-4 developed by somatic fusion with abrin had double the biomass, exo-polymer and cordycepin production as compared to both parental strains. In comparison the developed strains showed higher biomass, exo-polymer and cordycepin production (Fig. 3) rather the plasma fusion was not triggering biomass as well as metabolic production. The present study indicated that abrin is capable to develop 
strains with high metabolic products and biomass and will be a promising method for strain development in filamentous fungi.

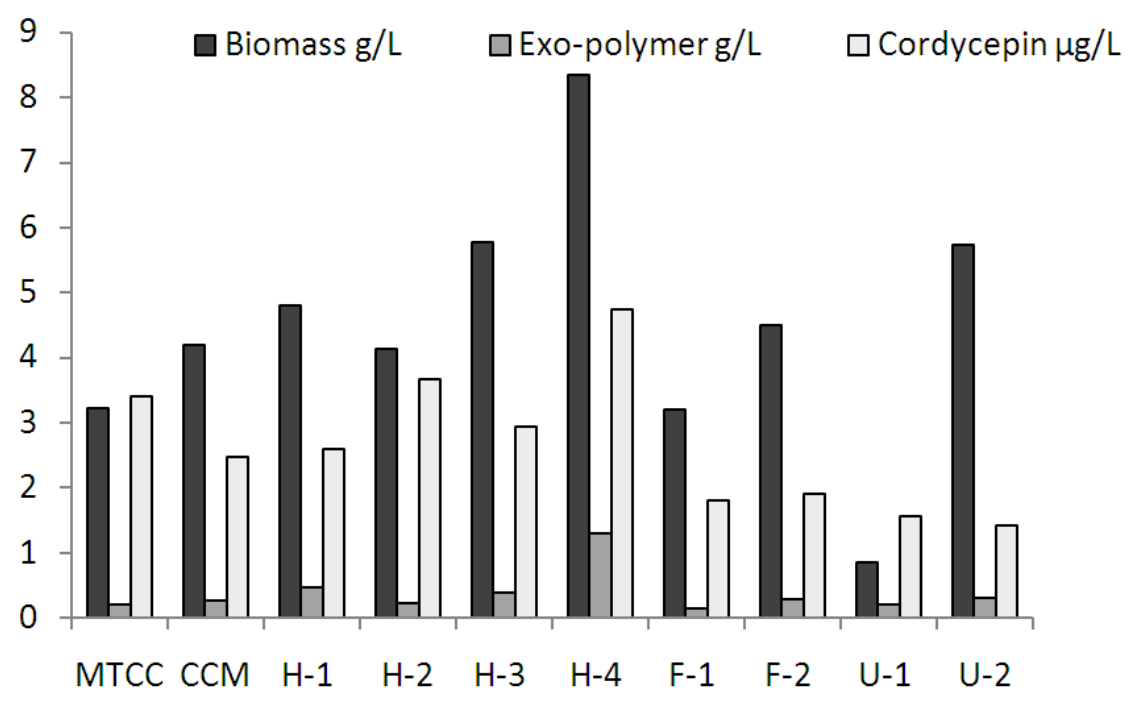

Fig. 3 - The biomass, exo-polymer and cordycepin produced by the improved cultures of C. militaris

\section{Discussion}

Shrestha et al. (2005) mated two strains of $C$. militaris in CMA medium and showed the potential improvent in metabolic production. The present study is based on the study conducted by Holliday et al. (2004) using Snake venom for somatic fusion of $C$. sinensis and the developed strains were reproducible strain and showed improved quantitative bioactive compounds. A similar effective somatic fusion was observed in the present study using abrin, a plant poison. The antifungal property of abrin isolated from A. precatorius has been reported earlier (Prashith et al. 2010, Roy et al. 2012, Bhatia et al. 2013). It was observed that abrin can inhibit the growth of $C$. militaris, rather aftersomatic fusion the strain the effect of abrin. It can be stated that $C$. militaris had somatic fusion due to the inhibitory property of abrin and future the fused colony survived the effect of abrin in the long time exposure.

The present study supports the approach conducted by Zhou\& Bian (2007) which showed increased cordycepin production and genetic stability in plasma fused strains of $C$. militaris. Moreover Guo et al. (2010) successfully fused $C$. sinensis and $C$. militaris and showed morphological and genetically similar to the parent strains. Moreover in both studies of plasma fusion heat and ultraviolet inactivation was done to stabilize the fusion but in the present study inactivation procedure was not followed to stabilize the fusion rather the fused suspension was directly inoculated to CMA from which the culture was isolated. It was observed that the isolated were stable even after sub-culturing and the colonies were having similar parental characteristics.

The use of low-energy ion beam in C. militaris can help to increase cordycepin production ( $\mathrm{Li}$ et al. 2009). Das et al. (2008) demonstrated that the proton beam irradiation increases the production of cordycepin and it was observed that the mutant had a superior production performance. The present study showed that UV irradiation can be used for strain development; rather this process produces mutants which should be completed evaluated as a new strain. Based on the study of Holliday et al. (2004) showcased the effectiveness the somatic fusion using snake venom in strain improvement, this study showed the effectiveness of abrin as a somatic fusion trigger.

\section{Conclusion}

The three techniques employed for strain alteration, were found to be effective in $C$. militaris. The hybrids produced were stable and showed improved the strains productivity. The present study shows that, the somatic fusion is more effective than plasma fusion. Moreover UV irradiation is a 
method of development of mutants and cannot be applied as an effective method. The protoplast fused strains were having similar parental characteristics. This study showed that the three techniques are effective in developing improved strain of $C$. militaris and can be further utilized to develop effective strains for metabolite and biomass production. The UV method had produced characteristic chances which were not stable and had produced mutants. The both fusion methods developed hybrids and were effective in producing quantitative higher biomass and metabolites. Since somatic fusion is an effective approach in strain alteration and abrin is a plant compound which can be easily extracted and are equally effective in fusion, makes it as an effective method of strain improvements in $C$. militaris. Moreover this approach can be evaluated in other fungal species.

\section{Acknowledgement}

This project was funded by Sarvajanik Jankalyan Parmarthik Nyas, Bhopal. We are grateful to Mr S.N. Vijaywargia, Chancellor of People's University and Ms Megha Vijawarghia, Director HR \& IT, People's University, for extending all the facilities for carrying out this study in Centre for Scientific Research and Development, People's University, Bhopal, India.

\section{References}

Alberts B, Johnson A, Lewis J, Raff M, Roberts K, Walter P. 2002 - Molecular biology of the cell, 4th edition New York: Garland Science.

Aswini L, Arunagirinathan N, Kavitha M. 2014 - Strain improvement of Pleurotus species by protoplast fusion. International J of Advancements in Res \& Technology 3, 32-38.

Bae JT, Sinha J, Park JP, Song CH, Yun JW. 2000 - Optimization of submerged culture conditions for exo-biopolymer production by Paecilomyces japonica. Journal of Microbiology Biotechnology $10,482-487$.

Bhatia M., Siddiqui NA, Sumeet G. 2013 - Abrus Precatorius (L.): An Evaluation of Traditional Bhatia Herb. Indo American Journal of Pharmaceutical Research 3, 3295-3315

Das SK, Masuda M, Hatashita M, Sakuraiand A, Sakakibara M. 2008 - A new approach for improving cordycepin productivity in surface liquid culture of Cordyceps militaris using high-energy ion beam irradiation. Letters in Applied Microbiology 47, 534-553

Das SK, Masuda M, Sakurai A. 2010 - Medicinal uses of the mushroom Cordyceps militaris: current state and prospects. Fitoterapia 81, 961-968.

Feng H, Sun Z, Li H, Qin P, Tang C, Fu R,Liu Y, Li P, Zheng A. 2012 - Preparation, purification and regeneration optimizing research of protoplasts from Rhizoctonia solani. African Journal of Microbiology Research 6, 3222-3230.

Guo CJ , Zhao R, Zhu WB. 2010 - Protoplast Fusion between Cordyceps sinensis and Cordyceps militaris. Food Science 31, 165-171.

Gupta U, Cheema GS, Sodhi HS, Phutela RP. 1997 - Protoplast isolation and regeneration in Agaricus bisporous strain MS 39. Mushroom Research 6, 59-62.

Holliday JC, Cleaver P, Loomis-Powers M, Patel D. 2004 - Analysis of quality and techniques for hybridization of medicinal fungus Cordycepssinensis (Berk.)Sacc. (Ascomycetes). International Journal of Medicinal Mushrooms 6, 151-164.

Zhou HY, YB Bian. 2007 - Identification and Selection of High Cordycepin-Yielding Protoplast Fusion Products of Cordyceps militaris. Acta Edulis Fungi 4, 69-74.

Li W, Zhao S, Chen H, Yuan H, Wang T, Huang X. 2009 - High-yielding cordycepin in Cordyceps militaris modified by low-energy ion beam. Chinese journal of biotechnology 25, 1725-1731.

Lin BQ, Li SP. 2011 - Cordyceps as an Herbal Drug. In: Benzie IFF, Wachtel-Galor S (eds) Herbal medicine: Biomolecular and clinical aspects, 2nd edition. CRC Press, Boca Raton, Florida 73105.

Patel KJ, Ingalhalli RS. 2013 - Cordyceps militaris (L.: Fr.) Link - An Important Medicinal Mushroom. Journal of Pharmacognosy and Phytochemistry 2, 315-319. 
Prashith KTR, Vinayaka KS, Soumya KV, Ashwini SK, Kiran R. 2010 - Antibacterial and Antifungal Activity of Methanolic Extract of Abrus pulchellus Wall and Abrus precatorius Linn- A Comparative Study. International Journal of Toxicological and Pharmacological Research 2, 26-29.

Rizwana R, Powell WA. 1992 - Ultraviolet Light-Induced Instability of Vegetative Compatibility Groups of Cryphonectria parasitica. Phytopathology 82, 1206-1211.

Roy S, Acharya R, Mandal NC, Barman S, Ghosh R, Roy R. 2012 - A comparative antibacterial evaluation of raw and processed Guñjā (Abrus precatorius Linn.) seeds. Ancient Science of Life $32,20-23$

Russell R, Paterson M. 2008 -Cordyceps - A traditional Chinese medicine and another fungal therapeutic biofactory? Phytochemistry 69, 1469-1495.

Shrestha B, Han SK, Yoon KS, Sung JM. 2005 - Morphological characteristics of conidiogenesis in Cordyceps militaris. Mycobiology 33, 69-76.

Wen T, Kang J, Hyde K D, Li G, Kang C, Chen X. 2014b - Phenotypic marking of Cordyceps militaris fruiting-bodies and their cordycepin production, Chiang Mai Journal of Science 41 846-857.

Wen T, Li G, Kang J, Kang C, Hyde KD. 2014a - Optimization of solid-state fermentation for fruiting body growth and cordycepin production by Cordyceps militaris, Chiang Mai Journal of Science $41858-872$

Zhao J, Chang ST. 1995 - Intra specific hybridization between Coprinuscinereus and Schizophyllum commune by PEG -induced protoplast fusion and electro-fusion. World Journal of Microbiology and Biotechnology 11, 585-590.

Zhao K, Sun L, Ma X, Li X, Wang X, Ping W, Zhou D. 2011 - Improved taxol production in Nodulisporiumsylviforme derived from inactivated protoplast fusion. African Journal of Biotechnology 10, 4175-4182.

Zhou X, Gong Z, Su Y, Lin J, Tang K. 2009 - Cordyceps fungi: natural products, pharmacological functions and developmental products. Journal of Pharmacy and Pharmacology 61, 279-291. 